\title{
10
}

\section{The Idea: Integrating Authoring Concepts for Mobile Agents into an Authoring Tool for Active Multimedia Mail}

\author{
Schirmer Jürgen, Kirste Thomas \\ Darmstadt Technical University \\ Interactive Graphics Systems Group. Telephone: +496151 155241. \\ Fax: +496151155 480. email: schirmer@zgdv.de \\ URL: http://zgdv.igd.fhg.de/
}

\begin{abstract}
Mobile agents are a competitive concept of client-server computing and are especially suitable in mobile environments, that are characterized by low bandwidth communication facilities and ad hoc connection/disconnection to stationary systems. They can be used for information retrieval and information filtering, in which case they evaluate replies and return only the relevant data. Mobile agents as a metaphor of active objects are created on a mobile device such as a Personal Digital Assistant (PDA), will be launched into the information galaxy and are fulfilling the mobile user's task on the services available on networked stationary systems. One transmission channel for these itinerant agents is email. This paper introduces ACTIVE $M^{3}$ as an example of an active mail framework, which can be regarded as a first approach in authoring mobile agents in a graphical interactive manner. ACTIVE $M^{3}$ integrates two known concepts: active mail and multimedia mail.

The idea of active or computational mail has existed since 1976 (3); transfer programs embedded in standard mail which automatically execute at the receivers site. This hiker -the active mail message- can also carry multimedia elements (audio, animation, image etc. and indeed sub-agents) in his backpack, which it can also render and orchestrate at the receivers site in a sophisticated manner. Therefore, synchronization concepts are introduced. To summarize, the objective of ACTIVE $M^{3}$ is the development and implementation of a system for the realization of active multimedia mail and a composing tool
\end{abstract}


as an easy to use interactive authoring environment. An extension towards a more generalized authoring environment for this remote programming concept enables authoring of mobile agents.

This paper introduces the basic ideas. The results of the proposed concept for a composer for active multimedia mail are presented through a realized prototype.

\section{Keywords}

Active Mail, Computational Mail, Multimedia Mail, MIME, Remote Programming, Mobile Agents, Itinerant Agents, Synchronization

\section{INTRODUCTION}

Essentially, active mail (6) simply means to send executable programs within a mail message, instead of passive data. On a somewhat more abstract level, active mail messages can be regarded as active objects traveling through the network searching for, distributing, and collecting information; interacting with receivers; making decisions based on context knowledge; replicating themselves, etc. Active mail is used for remote program installation $^{*}$, for initiating synchronous connections between several remote users without being intrusive (e.g., a collective writing facility)-so active mail could be a platform for Computer Supported Cooperative Work (CSCW)-, for survey generation and meeting scheduling etc.

Today active mail is also a suitable application in mobile environments characterized by low bandwidth communication facilities and small display devices. Active mails are launched every-time the user opens a wireless link to a stationary system. Afterwards these messages can hike between several heterogeneous locations and through different networks, collecting information, evaluating information, making transactions, etc. So indeed active mails are itinerant agents (9).

In conjunction with support for structured multimedia content data and user-interface toolkits, active mail messages could for instance build up a form on the receivers site, consisting of text fields (for editing purposes), multiple choice elements, anchor buttons for linking multimedia elements to the form, etc. accept and evaluate the answers of the receiver (e.g., check the consistency) and automatically send back a reply to the originator of the mail. The active part -the "hiker"- of the mail can also structure the contents of the backpack, the part of the mail containing multimedia elements, in a more sophisticated manner. Synchronization in time and space will be possible. We will call this enhanced version of active mail-active multimedia mail (7).

${ }^{*}$ E.g. updating the latest version of an application. 
To emphasize, elements of the backpack could be also sub-agents, which describe tasks that have to be synchronized in a certain manner. For instance the installation of a program as one task and the establishment of a persistent connection to a shared application as the task following at the end of the first task. An active mail message is basically a program written in a suitable interpreter language. At the receivers site, the interpreter is started automatically upon message access, executing the program contained in the message.

This of course implies, that creating an active mail message is equivalent to writing a program. The average user can, however, not be expected to do a programming job, when he simply wants to send a message to a friend or colleague. One of the challenges of active multimedia mail is therefore to create an authoring tool that makes the writing of active multimedia messages as simple as writing a standard text message.

\section{THE IDEA}

One step further towards a generalized authoring environment for asynchronous communication is the integration of visual specification technic for mobile agents. A novice user without programming knowledge wants to have a possibility to define his goals and needs in a graphical interactive manner.

A main feature of an agent is to help the user to accomplish his task. In a mobile scenario, in which the end-user has access to stationary systems over a wireless link via a portable computer, this task could for instance be, to find the cheapest offer for a hotel, to make a reservation, or to collect some technical product information, etc.

Today agent technologies are heavily discussed in the research community as well as in the commercial field. The most important instance of this new technology is characterized by the metaphor of a mobile agent. Basically mobile agents are programs, typically written in a script language, which may be dispatched from a client computer and transported to a remote server computer for execution. CORBA, HTTP and SMTP are possible transmission channels for mobile agents. SMTP or email is an adequate transmission channel concerning a mobile scenario. Every time the user connects to a stationary system, all outgoing messages inside the spool area will be delivered. Besides, all notifications and alert mechanisms to inform the end-user about any change in resource are based upon email exchange.

The advantage of this approach to integrate mobile agents into email is, that it is embedded into a communication environment, which is broadly available $\dagger$. Finally, the application domain for mobile agents like information finding/retrieval, information gath-

\footnotetext{
$\dagger_{\text {Email is ubiquitous. }}$
} 
ering/filtering, the update of programs, meeting scheduling, etc. is quite similar to the one suggested earlier for the concept of active mails.

\section{STATE OF THE ART: MOBILE AGENT TECHNOLOGY}

Agents have their origin in the research area of artificial intelligence (2). As mobile agents, they are suggested as a suitable enhancement of client server computing. A main feature as opposed to traditionally client server computing is, that the processing of queries (scripts) is asynchronous and does not depend upon a continuous link between two parties. A more precise definition of a mobile agent is given as follows: "A mobile agent is an encapsulation of program-code, data and execution state, which is able to migrate between networked computers autonomously and goal-oriented while executing."

There exists many variations in the literature concerning this definition. Migration are often reduced to transmission of program code (script) without execution state and includes unidirectional, bidirectional and multi-directional mobility. Autonomy and goalorientation are often related to the fact, that the agent acts on behalf of the author, that he has a user defined intention and strategy. Moreover mobile agents are suggested to be able to cooperate with their peers, that means that they are able to communicate and to negotiate. Intelligence skills of agents, the ability to learn, to plan and to inference, are also desirable for mobile agents. This implies the presence of a knowledge base and rule pattern, as well as an inference machine.

\subsection{Research activities}

Research activities in mobile agent technology focus on suitable infrastructures which support for instance the migration of agents, security aspects and the dynamical access of unclassified services (electronic commerce), etc. (see for instance (18) (1) (4)). There exists also a several suggestions to enrich general purpose languages towards mobility and security (AgentTCL (13), Java-To-Go (16), SafeTCL (7), etc.) . Research activities concerning communication skills of agents and knowledge sharing are done in the artificial intelligence domain.

The Knowledge-Sharing Effort of DARPA $\ddagger$ is developing an Agent Communication Language (ACL), which is known as Knowledge Query Manipulation Language (KQML) (12) and which constitutes a language based protocol for knowledge sharing for distributed inter-operation among knowledge-based systems. This effort is also developing the

$\ddagger_{\text {Defense Advanced Research Projects Agency }}$ 
Knowledge Interchange Format (KIF) (19), which means that knowledge of one agent can be represented in any language and can be transformed into KIF for exchange.

Research activities in the AI domain focus also on Agent Based System/Software Engineering (ABSE, ASE) $\S$.

It is a higher aggregated approach of object-technology with standardized message semantics, which was invented to facilitate the creation of software able to inter-operate, that is exchange information and services with other programs (in an expressive ACL) and thereby solve problems that cannot be solved alone. In this approach to software development, application programs are written as software agents, i.e. software components that communicate with their peers by exchanging messages in an expressive agent communication language.

Another research activity in the AI field are user interface agents. These are intelligent agents, which build an extension of the traditionally user-interface metaphor of direct manipulation towards the metaphor of indirect management. This indirect management is established by personal assistants, who are collaborating with the user and help to automate repetitious user tasks. The agents have the ability to observe the user behavior, to learn and to inference (e.g. information filtering, information retrieval, mail management, meeting scheduling etc. based on user preferences; intelligent help agents, that correct user mistakes; see also WebHunter at MIT (20)).

\subsection{The work carried out in the commercial field and the Internet}

\section{Information Retrieval and Filtering}

Due to rapid growing of the Internet and world wide web, technologies such as search engines are becoming well known and are often referred too as agents (spiders, robots, web-crawler, infobot etc.). These are search engines, which establish an index database after contacting web-servers in a synchronous fashion. A migratable executable script and real autonomy with inferencing capabilities does not exist.

Tacoma (24) is a research activity which extend WWW-servers to host itinerant agents, which perform information retrieval. As mentioned before WebHunter is an approach for information retrieval based on learning capabilities about user preferences.

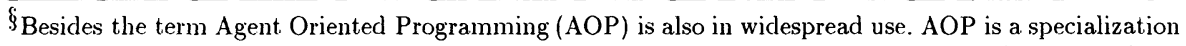
of Object-Oriented Programming (OOP). Agents are objects that have mental states (such as beliefs, desires and intentions) and a notion of time. They are programmed using commitment rules. These are simple forward chaining rules which connect messages, the agent's internal state and its actions. 


\section{Databases}

Oracle Mobile Agent (21) is an approach for client-agent-server communication. Mobility in regard to real transmission of scripts is not available; here, the only object which is mobile is the application domain and we can rather speak of static agents for mobile scenarios. The infrastructure consists of a message manager at the client side (a mobile device) and the agent event manager on the server side (or an the corporate LAN), which collects messages of the clients and passes them to application specific message handlers resp. transaction handler, which constitute the agent.

\section{Languages}

Java (23) is an example of embedding behavior into the World Wide Web, it brings HTML documents to life and visualizes information at the client site. Java do not support real mobility. Mobility is given through the capability to down-load programs (applets) from a server to a client (unidirectional). These applets can neither go any further nor can they go the other way round. Java addresses mainly security issues concerning the exchange of programs between heterogeneous networked systems. Recently there exists some research activities which enrich Java with communication and itinerant skills (see JavaToGo (16) and JavaAgentTemplate (15)).

A more sophisticated approach for a mobile agent language (and an electronic commerce scenario) is provided by General Magic's Magic Cap and Telescript (26) system. Telescript is an object-oriented language, which supports migration (statements: go, places, ticket, passport). This is an example of a well-designed electronic shopping system, which makes use of migratable mobile scripts, but suffers from the small amount of platforms be supported (MagicCap, Personal Link,UNIX) and the specific application domain (electronic shopping).

\subsection{Application Domain}

To conclude this section, a short impression of example application domains for mobile agents is given. Knowbots are stationary agents (e.g. launched from a mobile device, unidirectional), which implements a special kind of user profile (mail enabled application, dissemination of information, watchers, alert mechanisms for notification of any change in resource e.g. file size; personification of server behavior, etc.). The modeling of business processes as a collections of autonomous, problem solving agents which interact when they have interdependencies is an application domain for workflow-management systems (e.g. meeting scheduling). Mobile agents are suggested for distributed information retrieval resp. information filtering and data-retrieval. Mobile agent technology are also suitable for information dispersal, for instance to update the latest version of a program, and in computer supported cooperative work scenarios to establish a persistent connection to a 
groupware application. They are introduced to perform transactions in the database world and begin to gain attention in the financial world. A challenging domain in regard to the complexity of interaction, security aspects, trustworthiness, etc. is the electronic commerce field (electronic markets, markets for buying and selling goods, e.g. job, accommodation, car, flea-market, etc.; electronic shopping, open-bidding auction).

So far, the state of the art in regard to mobile agent technology has been introduced. Challenges have been identified. The introduction of a visual specification technic for mobile agents, which assists the novice user without programming knowledge in the definition of his goals and needs, is one challenging approach. The following chapter focuses on the development of a generalized authoring environment for mobile agents, that is currently being established. First of all, the already existing specialized authoring environment ACTIVE $M^{3}$ is introduced.

\section{THE ACTIVE $M^{3}$ COMPOSING TOOL}

The main goal of ACTIVE $M^{3}$ was to develop an easy-to use authoring system for active multimedia mail, based on the metaphor of direct manipulation.

The overall look-and-feel of the composing tool is derived from Graphical User Interface Builders (GUIB) like iX-Build: the most important aspect of an active mail message is the way it presents itself to the receiver - i.e., its user interface. Figure 1 gives an impression of what ACTIVE $M^{3}$ looks like.

The user creates an active message by interactively assembling message elements onto a mail form. This form may contain presentation/interaction elements such as buttons, textareas or in-line images. In addition, it may contain anchor-buttons, leading to multimedia content objects which are presented in external presentation tools, such as image, audio or video. Besides assembling active mails it is also possible to send normal email, linear multimedia mail, or active mails without a form-metaphor, etc. The construction and visualization of alternative mails is supported through the concept of different views.

After a message has been completed, ACTIVE $M^{3}$ converts the final form into a MIME(Multipurpose Internet Mail Extension) (5)-conform message. The active component of the message is represented as a LISP dialect (HyperPicture Command Language) (17) script contained in a special MIME-content part; multimedia objects are contained in another set of MIME-parts, the backpack (for in depth technical discussion of ACTIVE $M^{3}$ see (11)). 


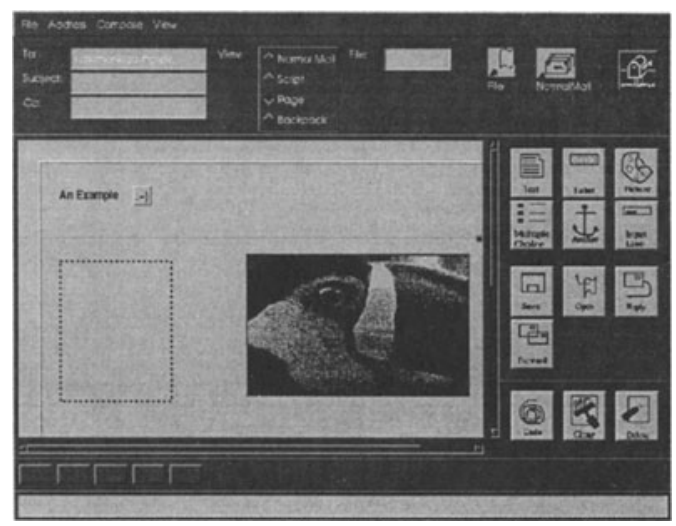

Figure 1 The user interface of ACTIVE $M^{3}$.

\subsection{Authoring the temporal structure of multimedia presentations}

As mentioned before, Active $M^{3}$ can describe interactive multimedia applications. These applications are characterized by the presentation of static and dynamic media which may be correlated in time and space. One possibility for instance is to use timed petrinets (Petri-Net Based Hypertext (22) and Object Composition Petri-Net (25)), directed weighted graphs, as the model for building synchronization interrelations among initially unrelated backpack objects. In our scenario the functionality of petri-nets to describe interrelations seems to be too powerful for the needs of a novice user, who may only want to send a little slide show about his recent holiday. Therefore, we suggest to establish basic synchronization descriptions with a subset of synchronization operators as proposed by the synchronization model of path-expressions (14). The selection of these operators and the selection of visible multimedia objects inside the backpack enables the interactive description of interrelationships among the data. A visualization is given by a directed graph (see Figure 2).

The reason for choosing path-expressions is that we need a user-level abstraction of the underlying concept of a finite-state-machine (used to implement synchronization), which 


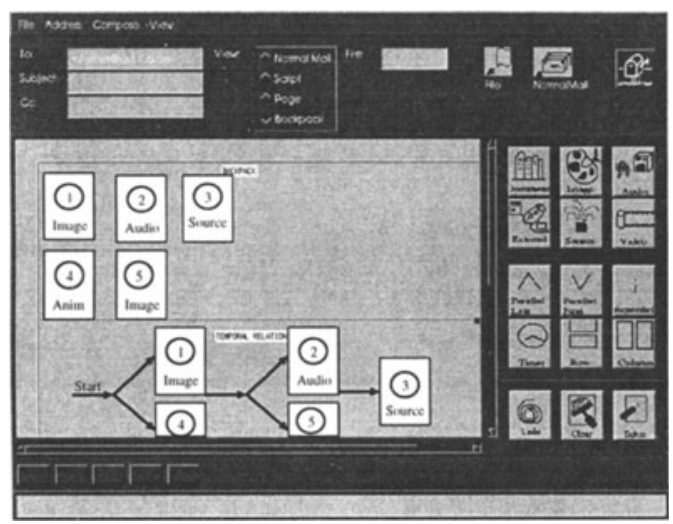

Figure 2 Multimedia inclusion and interactive description of synchronization with basic operators.

enables the adding of hand-crafted functionality in a very easy and intuitive manner in case the description power of the authoring mechanism is not sufficient.

\subsection{A Generalization towards Authoring of Mobile Agents}

We propose, that the construction of mobile agents is quite similar to the composition of active mails and the synchronization interrelation.

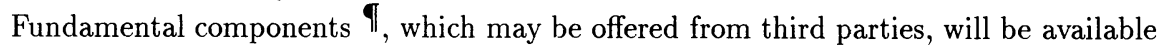
on a special panel and a workbench will be inserted into an additional view, in which the user can construct his own special agent and where he can get direct feedback of his construction.

An interesting approach in creating agents in a graphical interactive manner is given in the KidSim system (10). It is based on the concept of graphical rewrite rules and programming by demonstration. KidSim is an environment, which allows children to construct a

\footnotetext{
I For instance results of the componentware research. Componentware is an approach to create custom agents by combining components using scripts, which would contain domain specific expertise (e.q. financial planning) and would use the services of the components (text formatting, equation solving) to perform some of the actions.
} 
computer game. It has to be evaluated if the concept of programming by demonstration should be changed to programming by example in the context of mobile agents, because this is the way, people learn to program. Its additionally worthwhile to evaluate the concepts of visual programming (8) in this context. Proposed systems that are based on dataflow-diagramms seems to be not very intuitive for the needs of a mobile user (e.g. display-size of a PDA).

An agent is sometimes more than only an executable script. It has a mental state (beliefs, intention, goal), can communicate with other agents, and can inference to fulfill a defined task. So, agents are suitable for an open system, in which services available do not have to be known in advance. A novice user may visually program agents for his needs and goals, without concrete knowledge of existing services.

Consequently, the agent consists of a knowledge-base part and a behavior part. For the behavior part we may introduce a visual programming concept, but the user needs some additional mechanisms to construct the knowledge-base part. He (or the system) has to specify the knowledge of an agent in an appropriate knowledge representation language with a vocabulary (ontolingua) suitable for a special domain. This knowledge may also be derived and extended from a history of successfully performed tasks and user preferences.

The user may also wish to specify whether the agent should share his knowledge with other agents, add security properties, add electronic cash, add a list of locations to be visited or to be excluded. In addition he wants to specify the priority and the expiration time of the agent. To summarize, the user may wish to specify the degree of autonomy, intelligence, social ability, and mobility. Trustworthiness for such a system and the constructed agent is a very important attribute, and must be addressed as well as security aspects.

We conclude, that constructing a mobile agent for general purpose is a large design issue, for which influences of AI has to be regarded as well. ACTIVE $M^{3}$ is a first step and an appropriate basis towards such an effort.

\section{CONCLUSION AND FUTURE WORK}

In this paper, we have outlined the general concept of active multimedia mail. We then introduced a prototype composing tool for active messages, the ACTIVE $M^{3}$ tool. A user can develop "programs" without programming knowledge and it would be worthwhile to evaluate the concepts of visual programming and if they are well suited as the underlying metaphor of such a composing tool. As part of a Global Information System, ACTIVE $M^{3}$ can be regarded as an approach in generating mobile agents acting on behalf of the author in a graphical interactive manner. 


\section{ACKNOWLEDGMENT}

The synchronization concept and the work regarding mobile agents are performed in the MOVI project funded by the German Science Foundation (DFG).

\section{REFERENCES}

Intelligent Agents. Communications of the ACM, 37(7), July 1994.

R.H. Anderson and J.J. Gillogly. Rand intelligent terminal agent (rita): Design philosophy. Technical Memorandum R-1809-ARPA, Rand Corporation, February 1976.

Das Ara Projekt. Available via WWW

(URL: http://www.uni-kl.de/AG-Nehmer/Ara/ara_D.html).

Freed N. Borenstein, N. MIME (Multipurpose Internet Mail Extension). RFC 1521, IETF, 1993.

N. Borenstein. Computational mail as network infrastructure for computer supported cooperative work. In CSCW' '22, Toronto, 1992.

N.S. Borenstein. Email With A Mind of Its Own: The Safe-Tcl Language for Enabled Mail. In ULPAA'94, 1994. (also available as Internet Draft).

M.M. et al. Burnett. Visual Object-Oriented Programming. Manning Publications Co, Greenwich, 1995.

Colin Harrison David Levine Colin Parris Gene Tsudik David Chess, Benjamin Grosof. Itinerant Agents for Mobile Computing. IEEE Personal Communications, The Magazine of Nomadic Communication and Computing, 2(5), October 1995.

David Canfield Smith et al. KIDSIM: Programming Agents Without a Programming Language. Communications of the ACM, 37(7):55-67, July 1994.

J. Schirmer et al. ActiveM3 -An Authoring System for Active Multimedia Mail-. In Multimedia Computing and Networking 1996, volume 2667, January 1996.

Tim Finin et al. DRAFT: Specification of the KQML Agent-Communication Language. Technical report, DARPA Knowledge Sharing Initiative, June 1993.

Robert S. Gray. AgentTcl: A transportable agent system. In CIKM Workshop on Intelligent Information Agent, 4th International Conference on Information and Knowledge Management, Baltimore, Maryland, December 1995.

Petra Hoepner. Synchronizing the presentation of multimedia objects - oda extensions. In L.Kjelldahl, editor, Multimedia, Systems, Interaction and Applications (1st Eurographics Workshop, Stockholm, Sweden), pages 87-100. Springer-Verlag, 1991.

Java Agent Template, Version 2.0. Available via WWW

(URL: http://cdr.stanford.edu/ABE/JavaAgent.html). 
Java-To-Go: Itinerative Computing Using Java. Available via WWW (URL: http://ptolemy.eecs.berkeley.edu/ wli/group/java2go/java-to-go.html).

T. Kirste. HCL Language Reference Manual, Version 1.0. ZGDV-Report 68/93, 1993.

W. Lamersdorf M. Merz. Agents, Services and Electronic Markets: How do they Integrate? In to appear in: IFIP/IEEE International Conference on Distributed Platforms, Dresden, 1996.

Richard E. Fikes Michael R. Genesereth. Knowledge Interchange Format, Version 3.0, Reference Manual. Technical report, Computer Science Department, Stanford University, Stanford, California, June 1992.

WebHunter. Available via WWW

(URL: http://webhound.www.media.mit.edu/projects/webhound/doc/Webhound.html).

White Paper Oracle. Oracle Mobile Agents, Part A22547. Technical report, Oracle, March 1995.

P. David Stotts and Richard Furuta. Temporal Hyperprogramming. Journal of Visual Languages and Computing, pages 237-253, 1990.

SUN Microsystems, Mountain View, CA. The Java Language Specification Release 1.0 Alpha 2, March 1995.

TACOMA. Available via WWW (URL: http://www.cs.uit.no/DOS/Tacoma/Overview.html).

Arif Ghafoor Thomas D.C. Little. Synchronization and Storage Models for Multimedia Objects. IEEE Journal on Selected Areas in Communications, 8(3):413-427, 1990.

J.E. White. Telescript Technology: The Foundation for the Electronic Marketplace. Technical report, General Magic Inc, White Paper, 1994. 